\title{
Implementation of a Pharmacist-Led Culture and Susceptibility Review System in Urgent Care and Outpatient Settings
}

\begin{abstract}
Kristen N. Pierce, PharmD; Kim Clarke, PharmD; Marci M. Swanson, PharmD, BCACP; and Deborah Hobbs, PharmD
Background: Increasing prevalence of antibiotic resistance is an urgent public health threat. The purpose of this project is to implement a pharmacist-managed culture review service to decrease and prevent inappropriate use of antibiotics. This service will intervene in cases of mismatched antibiotic-bacteria combinations to decrease health care provider (HCP) and nursing interruptions, improve patient outcomes, and enhance prescribing practices to reduce occurrence of antibiotic resistance.

Observations: Patients requiring changes in antibiotic therapy after culture and susceptibility results were identified through the electronic health record. After results were returned, pharmacists assessed the antibiotic for appropriateness. If the isolated organism was not susceptible to the empiric antibiotic, the pharmacist adjusted the regimen,

counseled the patient, documented the intervention electronically, and notified the HCP via an electronic note. Follow-up phone calls assessed for adverse effects and answered patient questions. Pharmacists could change antibiotic therapy without contacting HCPs because of an antimicrobial stewardship care coordination agreement between HCPs and pharmacists. Previously, HCPs were responsible for evaluating culture and susceptibility results as well as adjusting antimicrobial regimens. After implementing this project, 10 interventions were made out of 320 patients from August 2019 to February 2020.

Conclusions: Appropriateness of antibiotic therapy through antimicrobial stewardship could help combat the significant public health issue of antibiotic resistance.
\end{abstract}

Author affiliations can be found at the end of the article. Correspondence:

Kristen Pierce

(kristen.pierce94@gmail.com)

Fed Pract. 2021;38(9). Published online September 12. doi:10.12788/fp.0173 ge ncreasing antibiotic resistance is an urgent threat to public health and establishing a review service for antibiotics could alleviate this problem. As use of antibiotics escalates, the risk of resistance becomes increasingly important. Each year, approximately 269 million antibiotics are dispensed and at least $30 \%$ are prescribed inappropriately. ${ }^{1}$ In addition to inappropriate prescribing, increased antibiotic resistance can be caused by patients not completing an antibiotic course as recommended or inherent bacterial mutations. According to the Centers for Disease Control and Prevention, each year approximately 3 million individuals contract an antibiotic-resistant infection. ${ }^{2}$ By 2050, it is projected that drug-resistant conditions could cause 300 million deaths and might be as disastrous to the economy as the 2008 global financial crisis. ${ }^{3}$ Ensuring appropriate use of antibiotic therapy through antimicrobial stewardship can help combat this significant public health issue.

Antimicrobial stewardship promotes appropriate use of antimicrobials to improve patient outcomes, reduce health care costs, and decrease antimicrobial resistance. One study found that nearly $50 \%$ of patients discharged from the emergency department with antibiotics required therapy modification after culture and susceptibility results were returned. ${ }^{4}$ Both the Infectious Disease Society of America (IDSA) and the Society for Healthcare Epidemiology of America (SHEA) support incorporating a clinical pharmacist into culture reviews. ${ }^{3}$ Several institutions have implemented a pharmacist-led culture review service to improve antibiotic usage, which has shown positive results. A retrospective case-control study at University of Rochester Medical Center showed reduced time to positive culture review and to patient or health care provider (HCP) notification when emergency medicine pharmacists were involved in culture review. ${ }^{5}$ A retrospective study at Carolinas Medical Center-Northeast showed 12\% decreased readmission rate using pharmacistimplemented culture review compared with HCP review. ${ }^{6}$ Results from previous studies showed an overall improvement in patient safety through decreased use of inappropriate agents and reduced time on inappropriate antibiotic therapy.

Establishing a pharmacist-led culture review service at the Carl Vinson Veterans Affairs Medical Center (CVVAMC) in Dublin, Georgia, could decrease the time to review of positive culture results, time to 
patient or HCP notification, and readmission rates. CVVAMC provides outpatient primary care services to about 30,000 veterans in the central and southern regions of Georgia. Our facility has executed an antimicrobial stewardship program based on guidelines published in 2016 by IDSA and SHEA to guide optimal use of antibiotics. Clinical pharmacists play an active role in antimicrobial stewardship throughout the facility. Clinical responsibilities of the antimicrobial stewardship pharmacist include assessing therapy for inappropriate dual anaerobic coverage, evaluating inpatient culture results within 48 hours, dosing and monitoring antibiotic therapy, including vancomycin and aminoglycosides, and implementing IV to bymouth conversions for appropriate patients. HCPs involved with antimicrobial stewardship could order an array of tests to assess a veteran's condition, including cultures, when an infection is suspected.

Culture results take about 3 to 5 days, then HCPs evaluate the result to ensure current antibiotic therapy is appropriate. Patients might not receive timely followup because HCPs often have many laboratory alerts to sift through every day, and a protocol is not in place for pharmacists to adjust outpatient antimicrobial regimens based on culture results. Before implementing this project, there was no outpatient service for pharmacists to impact culture and susceptibility review. This project was initiated because a lead physician identified difficulty reviewing culture and susceptibility results. HCPs often work on rotating schedules, and there was a concern about possible delay in followup of results if a HCP was not scheduled to work for a period of time.

The purpose of this project was to implement an outpatient, pharmacistmanaged culture and susceptibility review service to improve patient outcomes, including decreasing and preventing inappropriate antibiotic use. The primary objective was to design and implement a pharmacist-led review service to intervene in cases of mismatched antibiotic bacteria combinations. Secondary objectives included identifying most common culture types and organisms encountered and intervened on at our facility.
TABLE 1 Interventions Based on Antimicrobial Dashboard

\begin{tabular}{lc} 
Interventions & No. \\
\hline Veterans with pending culture results & 675 \\
\hline $\begin{array}{l}\text { Veterans with positive culture and an empiric } \\
\text { antibiotic prescribed }\end{array}$ & 320 \\
\hline Veterans requiring pharmacist intervention & 10 \\
\hline Antibiotics changed based on therapy mismatch & 7 \\
\hline $\begin{array}{l}\text { No antibiotic intervention made after contact by } \\
\text { clinical pharmacist: patient was asymptomatic }\end{array}$ & 3 \\
\hline
\end{tabular}

\section{QUALITY IMPROVEMENT PROJECT}

This quality improvement project was approved by the CVVAMC Pharmacy and Therapeutics Committee. Members of the medical review board signed a care coordination agreement between pharmacy and outpatient HCPs to permit pharmacist interventions involving optimization of antibiotic therapy. This agreement allowed pharmacists to make changes to existing antimicrobial regimens within their scope of practice (SOP) without requiring discussion with HCPs. A protocol was also developed to guide pharmacist modification of antimicrobial therapy based on current antimicrobial guidelines. ${ }^{7}$ This protocol was based on commonly isolated organisms and local resistance patterns and provided guidance for antibiotic treatment based on culture type (ie, skin and soft tissue infection, urine, etc). Computerized Patient Record System (CPRS) note templates were also developed for interventions performed, and patient follow-up after antibiotic regimens were completed (eAppendices 1 and 2 available at doi:10.12788/ fp.0173). HCPs were educated about the service through email and a flyer explaining the culture review process (eAppendix 3 available at doi:10.12788/fp.0173). This flyer was deemed sufficient for education because HCP responses generally were positive, and no additional education methods were requested. HCPs also seemed to view this intervention positively because the service aimed to reduce their burden.

\section{Program Inclusion}

Veterans were included in this project if they presented to primary care or urgent care 
TABLE 2 Antibiotic Therapy Interventions for Empiric Antibiotic/Pathogen Mismatches

\begin{tabular}{llll} 
Empiric Antibiotics & Pathogen-Directed Antibiotics & Culture Types & Isolated Organisms \\
\hline Sulfamethoxazole/trimethoprim & Cefdinir & Urine & Proteus mirabilis \\
\hline Cephalexin & Sulfamethoxazole/trimethoprim & Urine & Escherichia coli \\
\hline Sulfamethoxazole/trimethoprim & Ciprofloxacin & Urine & Pseudomonas aeruginosa \\
\hline Ciprofloxacin & Nitrofurantoin & Urine & Escherichia coli \\
\hline Ciprofloxacin & Cefdinir & Urine & Escherichia coli \\
\hline Levofloxacin & Cefdinir & Urine & Proteus mirabilis \\
\hline Levofloxacin & Nitrofurantoin & Urine & Enterococcus faecium
\end{tabular}

clinics for therapy; had positive culture and sensitivity results; and were prescribed an empiric antibiotic. Veterans were not eligible for this project if they were not receiving antibiotic therapy, with or without pending or resulted culture results shown in CPRS.

\section{Implementation}

Data gathered through a CPRS dashboard from August 2019 to February 2020 identified patients with pending or completed culture results in urgent care and primary care settings (eAppendix 4 available at doi:10.12788/fp.0173). The dashboard was created specifically for this project to show patient details that included initial antibiotic(s) prescribed and preliminary and final culture results. After a mismatched combination was identified, pharmacists contacted patients and assessed symptoms. If a patient was still symptomatic, the pharmacist changed the antibiotic regimen and educated the patient about this change. The pharmacist documented an intervention note in CPRS and added the HCP as a signer so he or she would be aware of the change. The clinical pharmacist followed up after regimens were complete. At this time, the pharmacist assessed patients to ensure the medication was taken as directed (eg, number of days of therapy, how many tablets per day, etc), to discuss any reported adverse effects, and to assess resolution of symptoms. If a patient still had symptoms, the pharmacist contacted the patient's primary care provider. If the veteran could not be contacted after 3 consecutive attempts via phone, a certified letter was mailed. If patients were asymptomatic at the time of the call, the pharmacist documented the lack of symptoms and added the HCP as a signer for awareness purposes. HCPs continued to practice as usual while this service was implemented.

\section{Observations}

Using the culture and susceptibility dashboard, the pharmacist identified 675 patients as having a pending culture (Table 1). Among these patients, 320 results were positive, and were taking antibiotics empirically. Out of the 320 patients who met inclusion criteria, 10 required pharmacist intervention. After contacting the veterans, 7 required regimen changes because their current antibiotic was not susceptible to the isolated organism. Three additional patients were contacted because of a mismatch between the empiric antibiotic and culture result. Antibiotic therapy was not modified because these patients were asymptomatic at the time the clinical pharmacist contacted them. These patient cases were discussed with the HCP before documenting the intervention to prevent initiation of unwarranted antibiotics.

Most of the modified antimicrobial regimens were found in urine cultures from symptomatic patients (Table 2 ). Of the 7 patients requiring therapy change because of a mismatch antibiotic-bacteria combination, 4 were empirically prescribed fluoroquinolones, 2 received levofloxacin, and 2 were prescribed ciprofloxacin. According to the most recent antibiogram at our 
facility, some organisms are resistant to fluoroquinolones, specifically Proteus mirabilis (P mirabilis) and Escherichia coli (E coli). These pathogens were the cause of urinary tract infections in 3 of 4 patients with fluoroquinolone prescriptions.

Through the CPRS dashboard, the pharmacist inadvertently identified 4 patients with positive culture results who were not on antibiotic therapy. These patients were contacted by telephone, and antibiotics were initiated for symptomatic patients after consultation with the HCP. The primary culture type intervened on was urine in 12 of 14 cases (86\%). The other 2 culture types included oropharynx culture (7\%) positive for an acute bacterial respiratory tract infection caused by group C Streptococcus and a stool culture (7\%) positive for Pseudomonas aeruginosa (P aeruginosa). E coli (36\%) was isolated in 5 cases and was the most commonly isolated organism. $P$ aeruginosa $(29 \%)$ was identified in 4 cases. Other organisms included $P$ mirabilis (14\%) in 2 patients and streptococcus species (14\%) in 2 cases. Enterococcus faecium (7\%) was isolated in 1 case.

\section{DISCUSSION}

This project was an innovative antimicrobial stewardship endeavor that helped initiate antibiotic interventions quickly and improve patient outcomes. The antimicrobial stewardship pharmacist independently performed interventions for patients without requiring HCP consultation, therefore decreasing HCP burden and possibly reducing time to assessment of culture results.

\section{Limitations}

The study results were limited due to its small sample size of antimicrobial interventions. The clinical pharmacist did not contact the patient when the antibiotic prescribed empirically by the HCP was appropriate for the isolated organism. Among the patients contacted, 3 were asymptomatic, did not require further antibiotic therapy, and no intervention was made. Provider education was deemed successful because HCPs did not request further information about the service. However, not all HCPs were provided education because of different shifts and inability to attend educational sessions. Closely work- ing with lead physicians within the facility provided an alternate method for information dissemination.

The care coordination agreement allowed the pharmacist to make changes if patients had a current prescription for an antibiotic. In addition to the changes to antibiotics, this project improved HCP awareness of culture results even in cases of symptomatic patients who were not prescribed therapy. When this occurred, the pharmacist contacted the patient to assess symptoms and then notified the HCP if the patient was symptomatic.

\section{Future Directions}

Future endeavors regarding this project include modifying the scope of the service to allow pharmacists to prescribe antibiotics for patients with positive cultures and symptoms without empiric antibiotics in addition to continuing to modify empiric therapy. Additionally, improving dashboard efficiency through changes to include only isolated antibiotic mismatches rather than all antibiotics prescribed and all available cultures would reduce the pharmacists' time commitment. Expanding to other parts of the medical center, including long-term care facilities and other outpatient clinics, would allow this service to reach more veterans. Integrating this service throughout the medical center will require continued HCP education and modifying care coordination agreements to include these facilities.

On a typical day, 60 to 90 minutes were spent navigating the dashboard and implementing this service. The CPRS dashboard should be modified to streamline patients identified to decrease the daily time commitment. Re-education of HCPs about resistance rates of fluoroquinolones and empirically prescribing these agents also will be completed based on empiric antibiotic interventions made with these agents throughout this project. Discussing HCP viewpoints on this service would be beneficial to ensure HCP satisfaction.

\section{CONCLUSIONS}

This pharmacy service and antimicrobial stewardship program reduced time patients were on inappropriate antibiotics. Pharmacists reviewed the dashboard daily under 
the scope of this project, which expedited needed changes and decreased provider burden because pharmacists were able to make changes without interrupting HCPs' daily tasks, including patient care.

This program may also reduce readmissions. Patients who were still symptomatic were contacted could be given revised medication regimens without the patient returning to the facility for follow-up treatment. An interesting conclusion not included in the current scope of this service was possible reduced time to therapy initiation in cases of positive cultures and symptomatic patients without antibiotic therapy. If this occurred on the dashboard, patient's symptoms could be assessed, and if symptoms were ongoing, the pharmacist contacted the HCP with a recommended antimicrobial therapy. In these cases, we were able to mail the antibiotic quickly, and many times, on the same day as this intervention through overnight mail. Implementation of a pharmacist-led antimicrobial review service has provided positive results overall for CVVAMC.

\section{Acknowledgment}

This material is the result of work supported with resources and the use of the facilities at the Carl Vinson VA Medical Center.

\section{Author affiliations}

Kristen Pierce is a Clinical Pharmacy Specialist at St. Joseph's/Candler Health System in Savannah, Georgia. At the time the article was written she was a PGY-1 Pharmacy Resident at the Carl Vinson Veterans Affairs Medical Center in Dublin, Georgia. Kim Clarke is a Clinical Pharmacy Specialist, Acute Care/Antimicrobial Stewardship; Marci Swanson is a Clinical Pharmacist Practitioner and the PACT Clinical Pharmacy Supervisor and PGY-1 Pharmacy Residency Coordinator; Deborah Hobbs is the Associate
Chief of Clinical Pharmacy Services and PGY-1 Pharmacy Residency Director; all at the Carl Vinson Veteran Affairs Medical Center.

\section{Author disclosures}

The author reports no actual or potential conflicts of interest with regard to this article.

\section{Disclaimer}

The opinions expressed herein are those of the authors and do not necessarily reflect those of Federal Practitioner, Frontline Medical Communications Inc., the US Government, or any of its agencies. This article may discuss unlabeled or investigational use of certain drugs. Please review the complete prescribing information for specific drugs or drug combinations-including indications, contraindications, warnings, and adverse effectsbefore administering pharmacologic therapy to patients.

\section{References}

1. Centers for Disease Control and Prevention. Antibiotic use in outpatient settings, 2017: progress and opportunities. Accessed August 19, 2021. https://www.cdc.gov /antibiotic-use/stewardship-report/outpatient.html

2. Centers for Disease Control and Prevention. Antibiotic/antimicrobial resistance. Accessed August 19, 2021. https:// www.cdc.gov/drugresistance/index.html

3. Jonas OB, Irwin A, Berthe FCJ, Le Gall FG, Marquez PV. Drug-resistant infections: a threat to our economic future. March 2017. Accessed August 19, 2021. https:// documents.worldbank.org/en/publication/documents -reports/documentdetail/323311493396993758/final -report

4. Davis LC, Covey RB, Weston JS, Hu BBY, Laine GA. Pharmacist-driven antimicrobial optimization in the emergency department. Am J Health Syst Pharm. 2016;73(5) (suppl 1):S49-S56. doi:10.2146/sp150036

5. Baker SN, Acquisto NM, Ashley ED, Fairbanks RJ, Beamish SE, Haas CE. Pharmacist-managed antimicrobial stewardship program for patients discharged from the emergency department. J Pharm Pract. 2012;25(2):190194. doi:10.1177/0897190011420160

6 Randolph TC, Parker A, Meyer L, Zeina R. Effect of a pharmacist-managed culture review process on antimicrobial therapy in an emergency department. Am J Health Syst Pharm. 2011;68(10):916-919. doi:10.2146/ajhp090552

7. Infectious Diseases Society of America. Infectious diseases society of America guidelines 2019. Accessed August 24, 2021. https://www.idsociety.org/practice -guideline/practice-guidelines/\#/+/0/date_na_dt/desc 